Journal of Engineering and Applied Sciences 14 (16): 5762-5768, 2019

ISSN: 1816-949X

(C) Medwell Journals, 2019

\title{
A Digital Image Watermarking Scheme based on Discrete Cosine Transform
}

\author{
Ghazwan Jabbar Ahmed, Adel Jalal Yousif and Fadhil Kadhim Zaidan \\ Electronic Computing Center, University of Diyala, Diyala, Iraq
}

\begin{abstract}
In this research, a grayscale image watermarking scheme is proposed using a Discrete Cosine Transform (DCT) to embed a binary invisible watermark image. The DCT is applied to the entire host image instead of dividing the image into $8 \times 8$ non-overlapping blocks as usual in the conventional schemes and then some of the coefficients in the low-frequency DCT band are selected for the watermark embedding process. For obtaining an acceptable trade-off between the robustness and imperceptibility of the watermark, the watermarking strength is controlled by a robustness factor. The value of the robustness factor is not threshold and can be changed by the user based on the required robustness level and the characteristics of the host image. Peak Signal to Noise Ratio (PSNR) and Normalized Correlation (NC) are calculated to evaluate the images quality. The simulations and results demonstrate that the proposed scheme still holds its validity under various attacks such as JPEG compression, low-pass filtering and noise attacks.
\end{abstract}

Key words: Discrete cosine transform, digital watermarking, copyright protection, JPEG compression, watermarking techniques, normalized correlation, robustness and imperceptibility

\section{INTRODUCTION}

With the growing development of computers and network technologies, digital multimedia such as image, audio and video can now be created and distributed through the internet simply and quickly. Copyright protection has become a challenging issue due to the possibility of illegal alteration and unlimited copying. One of the most effective solutions is the use of digital watermarking techniques. Digital watermarking refers to the process of embedding some information (which is known as watermark) into the digital multimedia content in some way, so that, it can be extracted or identified later for various purposes such as content authentication, copyright protection, data integrity detection, ownership verification, etc. (Abdullatif et al., 2013).

Depending on human perception, the digital watermarking can be classified into two main categories namely, visible watermarking and invisible watermarking, the second category is considered in this research. In case of visible watermarking, the inserted watermark is intentionally visible to the human eye in the host image to show some important information such as TV channel logo or company logo. Whereas the invisible watermark is embedded in a secret location and can be extracted only by authorized persons in such a way preserving the perceptual content of the watermarked image similar to the original image (Yusnita and Khalifa, 2007; Giri et al., 2015).

The essential parameters that determine the efficiency of the invisible watermarking scheme are robustness and imperceptibility. The robustness of a watermark can be defined as its ability to resist any unintentional attacks such as signal processing operations or intentional attacks. While the imperceptibility of a watermark means that the difference between the watermarked and the original images should be unnoticeable by human eyes (Huynh-The et al., 2016). The digital watermarking can be implemented by spatial domain or transform domain. The principle of spatial domain algorithms is to embed a watermark into an image by directly altering the values of certain pixels in the cover image (Islam and Chong, 2014). Whereas in the transform domain, the watermark is embedded into the transformed coefficients of the host image by using the transformed algorithms such as Discrete Cosine Transform (DCT) (Kumar and Anuradha, 2014), Discrete Wavelet Transform (DWT) (Gunjal and Mali, 2011) or other transform domain algorithm. The transform domain techniques have several advantages over the spatial domain techniques. It has more robust watermarking and more control of imperceptibility which make it an attractive choice as demonstrated by various surveys (Sinha et al., 2014; Parashar and Singh, 2014; Malshe et al., 2012). In this research, the transform domain is employed in the design of the proposed watermarking technique by using the DCT algorithm.

\section{MATERIALS AND METHODS}

Discrete cosine transform: Discrete Cosine Transform (DCT) is basically an orthogonal transformation which is

Corresponding Author: Ghazwan Jabbar Ahmed, Electronic Computing Center, University of Diyala, Diyala, Iraq 


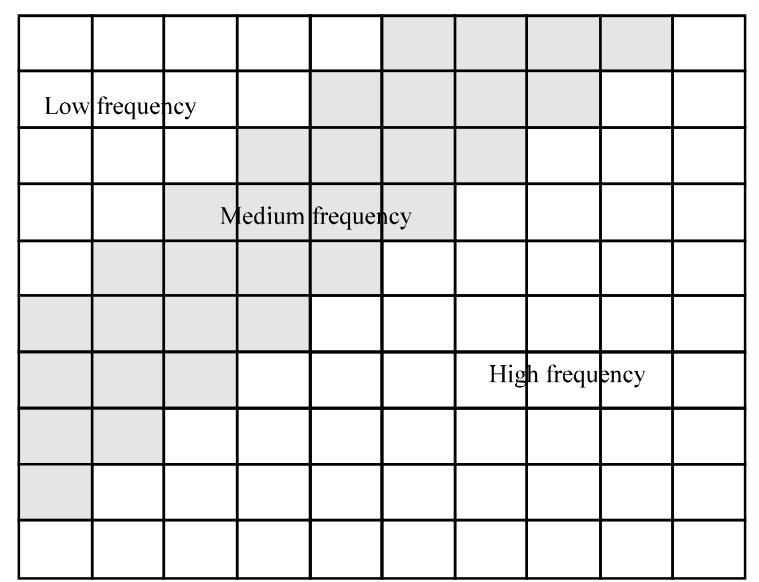

Fig. 1: DCT bands regions

used to transform a signal from the spatial domain to the frequency domain. The essential feature of the DCT is to collect the vast majority of the signal information in a few low-frequency components and ignoring the part that the human eye is least sensitive to. Additionally, DCT has a number of good features that make it one of the most commonly used tools in transformation domain such as strong energy compaction, moderate of complexity, less bit error rate and others (Ram, 2013; Eswaraiah et al., 2012). Thus, DCT tends to reduce the signal information by removing the redundant data of the transformed signal. Generally, the DCT divide the image into three different bands of frequencies namely low, medium and high-frequency band as shown in Fig. 1. The coefficient in the top-left represents the DC component and the others stand for AC components (Santhi and Thangavelu, 2011; Singh et al., 2013). In this research, the watermark is inserted in the low-frequency band to increase the robustness of the watermarking scheme (Mishra et al., 2015; Chen and Huang, 2008). For an image of size $(M \times N)$, the 2-Dimensional Discrete Cosine Transform (2D-DCT) and Inverse Discrete Cosine Transform (2D-IDCT) are defined as follows (Chen and Huang, 2008):

$$
\begin{gathered}
F(u, v)=c(u) c(v) \sum_{\mathrm{z}=0}^{\mathrm{M}-1} \sum_{\mathrm{y}=0}^{\mathrm{N}-1} \mathrm{f}(\mathrm{x}, \mathrm{y}) \cos \frac{\pi(2 \mathrm{x}+1)}{2 \mathrm{M}} \cos \frac{\pi(2 \mathrm{y}+1)}{2 \mathrm{~N}} \\
\mathrm{c}(\mathrm{u})=\left\{\begin{array}{cc}
\frac{\sqrt{1 / \mathrm{M}}}{\sqrt{2 / \mathrm{M}}} & \mathrm{u}=1,2,3, \ldots, \mathrm{M}
\end{array}\right. \\
v=0 \\
\mathrm{c}(v)=\left\{\begin{array}{l}
\sqrt{1 / \mathrm{N}} \\
\sqrt{2 / \mathrm{N}}
\end{array} v=1,2,3, \ldots, \mathrm{N}\right.
\end{gathered}
$$

$$
\mathrm{f}(\mathrm{x}, \mathrm{y})=\sum_{\mathrm{u}=0}^{\mathrm{M}-1} \sum_{\mathrm{v}=0}^{\mathrm{N}-1} \mathrm{c}(\mathrm{u}) \mathrm{c}(v) \mathrm{F}(\mathrm{u}, v) \cos \frac{\pi(2 \mathrm{x}+1) \mathrm{u}}{2 \mathrm{M}} \cos \frac{\pi(2 \mathrm{y}+1) v}{2 \mathrm{~N}}
$$

Proposed scheme: In this research, a watermarking scheme is proposed to insert a binary watermark image into a grayscale image in transform domain by using the DCT algorithm. The DCT is performed to the entire image and the low-frequency band is employed for embedding the binary bits of the watermark by choosing the most significant coefficients excluding the DC term which is located at the position $(0,0)$ in the coefficients matrix. The algorithms of watermark embedding and extraction are presented below.

Watermark embedding algorithm: The procedure of the watermarking embedding process is summarized by the following steps:

- Reading the original grayscale image $f$ with the size $\mathrm{M} \times \mathrm{N}$ and the binary watermark image with the size $\mathrm{q} \times \mathrm{r}$

- Performing the DCT to the entire $\mathrm{M} \times \mathrm{N}$ image $\mathrm{f}$ to obtain the coefficients matrix denoted by $\mathrm{F}$

- Choosing the (n) maximum coefficients $\mathrm{C}_{\max }=\left\{\mathrm{c}_{1}, \mathrm{c}_{2}\right.$, $\left.\ldots, c_{n}\right\}$ from the matrix $F$ excluding the DC term where: $\mathrm{n}=\mathrm{q} \times \mathrm{r}, \mathrm{C}_{\max } \in \mathrm{F}$

- Embedding an element $W(i, j)$ of the watermark using the chosen coefficients of $\mathrm{C}_{\max }$ by applying the following Eq. 5:

$$
\mathrm{c}_{\mathrm{k}}^{\prime}=\mathrm{c}_{\mathrm{k}} *(1+\mathrm{t} * \delta)
$$

where, $c^{\prime}{ }_{k}$ and $c_{k}$ represent the watermarked and the original coefficients, respectively. $\mathrm{K}=1,2,3, \ldots, \mathrm{n}$ :

$$
t=\left\{\begin{array}{l}
1, W(i, j)=0 \\
-1, W(i, j)=1
\end{array}\right.
$$

where, $1 \leq i \leq \mathrm{q}, \quad 1 \leq \mathrm{j} \leq \mathrm{r}, \delta$ represents the watermarking strength which is discussed. Performing the inverse DCT to the modified matrix coefficients to obtain the watermarked image. Figure 2 illustrates the watermark embedding algorithm.

Watermark extraction algorithm: The procedure of the watermarking extraction process is summarized by the following steps: Reading the watermarked $\mathrm{f}^{\prime}$ image and also the original image f. Performing the DCT to the entire watermarked image $\left(f^{\prime}\right)$ and the original image (f) to produce the coefficients matrices $\mathrm{F}^{\prime}$ and $\mathrm{F}$, respectively. Selecting the (n) watermarked DCT coefficients $\mathrm{C}^{\prime}{ }_{\mathrm{w}}=$ $\left\{\mathrm{c}_{\mathrm{w} 1}^{\prime}, \mathrm{c}_{\mathrm{w} 2}^{\prime}, \ldots, \mathrm{c}^{\prime}{ }_{\mathrm{wn}}\right\}$ from $\mathrm{F}^{\prime}$ that have the same position of the original DCT coefficients $\mathrm{C}_{\max }=\left\{\mathrm{c}_{1}, \mathrm{c}_{2}, \ldots, \mathrm{c}_{\mathrm{n}}\right\}$ in matrix: 


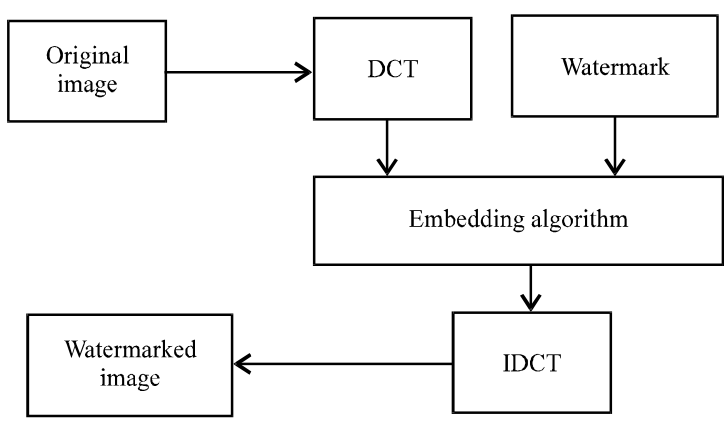

Fig. 2: Watermark embedding algorithm

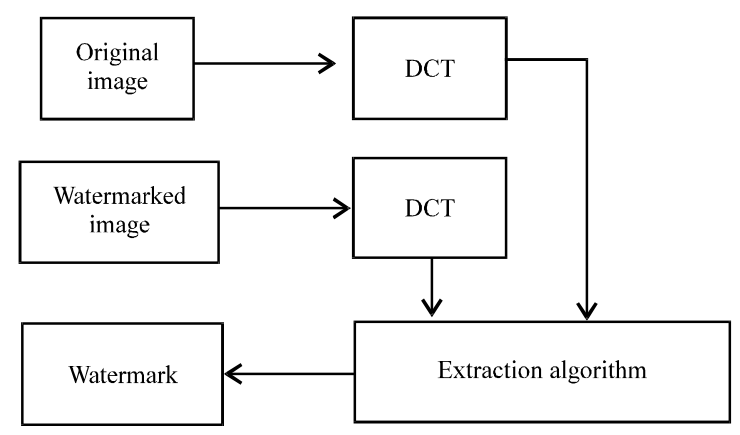

Fig. 3: Watermark extraction algorithm

$$
\text { let } \mathrm{d}_{\mathrm{k}}=\left(\mathrm{c}_{\mathrm{wk}}^{\prime} / \mathrm{c}_{\mathrm{k}}\right)-1
$$

where, $\mathrm{k}=1,2,3, \ldots, \mathrm{n}$. Extracting the watermark $\mathrm{W}^{\mathrm{e}}$ by making the judgment of $\mathrm{d}_{\mathrm{k}}$ :

$$
W^{e}(i, j)=\left\{\begin{array}{c}
0, d_{k}>0 \\
1, d_{k} \leq 0
\end{array}\right.
$$

where, $1 \leq i \leq \mathrm{q}, 1 \leq \mathrm{j} \leq \mathrm{r}$. Figure 3 illustrates the watermark extraction algorithm.

\section{RESULTS AND DISCUSSION}

The performance of the watermarking techniques is usually evaluated by measuring both properties of robustness and imperceptibility. In this study, 3 widely known grayscale images: Boats, Baboon and Goldhill with the size of $(512 \times 512)$ are taken to be the cover images for inserting binary watermark image with the size of $(30 \times 40)$ as shown in Fig. 4.

The robustness of the proposed watermarking scheme is evaluated by analyzing the watermarking strength under the commonly used signal processing operations such as JPEG compression with various Quality Factors $(\mathrm{QF})$ and low-pass filtering in addition to the noise attacks. The Normalized Correlation (NC)

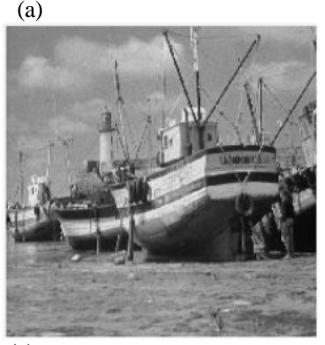

(c)

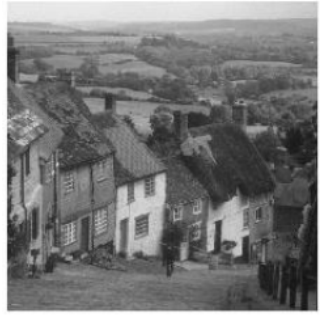

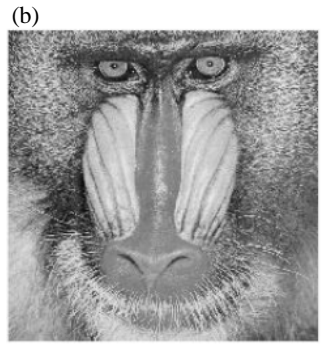

(d)

\section{LOGD}

Fig. 4: Test images; a) Boats; b) Baboon; c) Goldhill and d) Watermark

criterion is used to estimate the watermark robustness by calculating the degree of similarity between the original watermark $(\mathrm{W})$ and the extracted one $\mathrm{W}^{e}$. When the value of the normalized correlation is 1 , the extracted watermark is exactly like the original one. This value decreases as the difference between the two images increases as defined by the following Eq. 9, (Jawad et al., 2014):

$$
N C=\frac{\sum_{i=1}^{\mathrm{q}} \sum_{j=1}^{\mathrm{r}}\left(\mathrm{W}(\mathrm{i}, \mathrm{j}) \times \mathrm{W}^{\mathrm{e}}(\mathrm{i}, \mathrm{j})\right)}{\sum_{\mathrm{i}=1}^{\mathrm{q}} \sum_{\mathrm{j}=1}^{\mathrm{r}}(\mathrm{W}(\mathrm{i}, \mathrm{j}))^{2}}
$$

The imperceptibility of the proposed watermarking scheme is evaluated by measuring the visual quality of the watermarked image by using the Peak Signal to Noise Ratio (PSNR) criterion. The high value of PSNR means high level of watermark imperceptibility in the cover image. The following equation describes the PSNR (Jawad et al., 2014):

$$
\text { PSNR }=10 \log _{10}\left(\frac{225^{2}}{\mathrm{MSE}}\right)
$$

where, MSE is given by:

$$
\operatorname{MSE}=\frac{\sum_{\mathrm{x}=1}^{\mathrm{M}} \sum_{\mathrm{y}=1}^{\mathrm{N}}\left(1(\mathrm{x}, \mathrm{y})-\mathrm{l}^{\prime}(\mathrm{x}, \mathrm{y})\right)}{\mathrm{M} \times \mathrm{N}}
$$

In general, the robustness and imperceptibility requirements affect each other in reverse. In other words, 
the high robustness watermarking can be achieved but the imperceptibility will be degraded due to the added signal and vice versa. Therefore, watermarking techniques should balance between the robustness and imperceptibility requirements. In this research, the watermarking strength is controlled by a factor $(\delta)$ which is represent the robustness factor to achieve the required balance between the robustness and imperceptibility. So, high level of robustness can be achieved by increasing the value of $(\delta)$ but at the same time the watermarked image quality will be decreased and vice versa. In addition, the value of $(\delta)$ can be changed by the user based on the desired robustness level and the characteristics of the host image. The effect of changing the robustness factor value on the watermarked boats image is shown visually in Fig. 5.

Table 1 and 2 show arithmetically the effect of changing the value of on the performance of the proposed
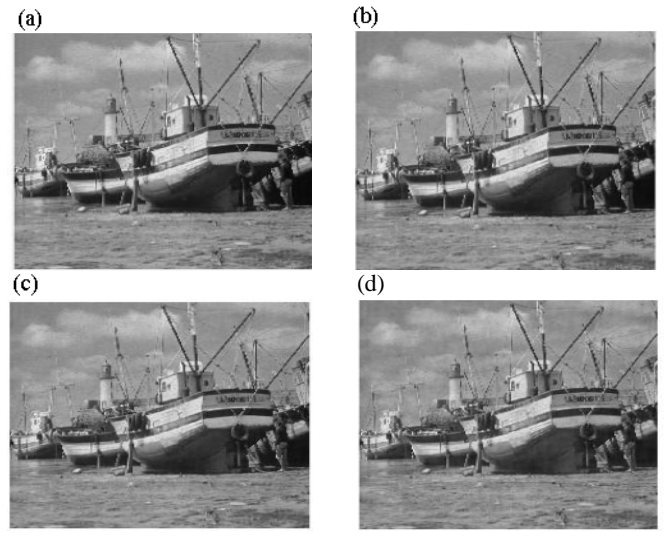

Fig. 5: a) Original boats image; b) $\delta=0.05$, PSNR $=41.53$; c) $\delta=0.1$, PSNR $=35.51$ and d) $\delta=0.2$, PSNR $=29.49$ watermarked Boats image with different value of $\delta$

Table 1: Performance analysis with low-pass filtering and JPEG compression attacks

\begin{tabular}{|c|c|c|c|c|c|c|c|c|c|c|c|c|}
\hline \multirow[b]{2}{*}{ Images $/ \delta$} & \multicolumn{2}{|c|}{ No attack } & \multicolumn{2}{|c|}{$\begin{array}{l}\text { Average filter } \\
(3 \times 3)\end{array}$} & \multicolumn{2}{|c|}{$\begin{array}{l}\text { Median filter } \\
(3 \times 3)\end{array}$} & \multicolumn{2}{|c|}{$\begin{array}{l}\text { JPEG }(Q F=20) \\
\text { compression }\end{array}$} & \multicolumn{2}{|c|}{$\begin{array}{l}\mathrm{JPEG}(\mathrm{QF}=40) \\
\text { compression }\end{array}$} & \multicolumn{2}{|c|}{$\begin{array}{l}\text { JPEG }(Q F=60) \\
\text { compression }\end{array}$} \\
\hline & PSNR & $\mathrm{NC}$ & PSNR & $\mathrm{NC}$ & PSNR & $\mathrm{NC}$ & PSNR & $\mathrm{NC}$ & PSNR & $\mathrm{NC}$ & PSNR & $\mathrm{NC}$ \\
\hline \multicolumn{13}{|c|}{ Boats } \\
\hline 0.01 & 55.51 & 1.0000 & 28.84 & 0.9137 & 30.95 & 0.7108 & 30.47 & 0.6155 & 32.72 & 0.7152 & 34.16 & 0.7522 \\
\hline 0.05 & 41.53 & 1.0000 & 28.59 & 0.9742 & 30.64 & 0.9507 & 30.15 & 0.8890 & 32.20 & 0.9798 & 33.46 & 1.0000 \\
\hline 0.1 & 35.51 & 1.0000 & 27.95 & 0.9798 & 29.73 & 0.9966 & 29.29 & 0.9776 & 30.91 & 1.0000 & 31.79 & 1.0000 \\
\hline 0.15 & 31.99 & 1.0000 & 27.07 & 0.9798 & 28.53 & 1.0000 & 28.15 & 0.9978 & 29.34 & 1.0000 & 29.96 & 1.0000 \\
\hline 0.2 & 29.49 & 1.0000 & 26.09 & 0.9854 & 27.25 & 1.0000 & 26.95 & 1.0000 & 27.82 & 1.0000 & 28.23 & 1.0000 \\
\hline \multicolumn{13}{|l|}{ Baboon } \\
\hline 0.01 & 56.71 & 1.0000 & 22.36 & 0.9070 & 22.85 & 0.4888 & 24.64 & 0.6031 & 26.85 & 0.6883 & 28.70 & 0.7175 \\
\hline 0.05 & 42.73 & 1.0000 & 22.32 & 0.9552 & 22.84 & 0.7231 & 24.58 & 0.8722 & 26.74 & 0.9854 & 28.54 & 0.9955 \\
\hline 0.1 & 36.71 & 1.0000 & 22.20 & 0.9664 & 22.75 & 0.8890 & 24.39 & 0.9776 & 26.43 & 0.9989 & 28.07 & 1.0000 \\
\hline 0.15 & 33.18 & 1.0000 & 22.01 & 0.9709 & 22.58 & 0.9496 & 24.08 & 0.9966 & 25.95 & 1.0000 & 27.38 & 1.0000 \\
\hline 0.2 & 30.69 & 1.0000 & 21.76 & 0.9753 & 22.34 & 0.9753 & 23.68 & 1.0000 & 25.36 & 1.0000 & 26.58 & 1.0000 \\
\hline \multicolumn{13}{|l|}{ Goldhill } \\
\hline 0.01 & 53.91 & 1.0000 & 29.76 & 0.9316 & 31.62 & 0.6323 & 30.74 & 0.5975 & 32.83 & 0.6771 & 34.19 & 0.7007 \\
\hline 0.05 & 39.93 & 1.0000 & 29.37 & 0.9563 & 31.18 & 0.9395 & 30.36 & 0.8475 & 32.21 & 0.9630 & 33.35 & 0.9933 \\
\hline 0.1 & 33.91 & 1.0000 & 28.45 & 0.9675 & 29.97 & 0.9865 & 29.27 & 0.9686 & 30.69 & 0.9989 & 31.46 & 1.0000 \\
\hline 0.15 & 30.39 & 1.0000 & 27.29 & 0.9742 & 28.48 & 0.9966 & 27.94 & 0.9955 & 28.93 & 1.0000 & 29.43 & 1.0000 \\
\hline 0.2 & 27.89 & 1.0000 & 26.07 & 0.9776 & 26.98 & 0.9989 & 26.60 & 1.0000 & 27.28 & 1.0000 & 27.61 & 1.0000 \\
\hline
\end{tabular}

Table 2: Performance analysis with different noise attacks

\begin{tabular}{|c|c|c|c|c|c|c|c|c|c|c|c|c|}
\hline \multirow[b]{2}{*}{ Images $/ \delta$} & \multicolumn{2}{|c|}{$\begin{array}{l}\text { Gaussian noise } \\
(\mu=0, v=0.001)\end{array}$} & \multicolumn{2}{|c|}{$\begin{array}{l}\text { Gaussian noise } \\
(\mu=0, v=0.005)\end{array}$} & \multicolumn{2}{|c|}{$\begin{array}{l}\text { Salt and pepper } \\
(0.5 \%)\end{array}$} & \multicolumn{2}{|c|}{$\begin{array}{l}\text { Salt and pepper } \\
(1 \%)\end{array}$} & \multicolumn{2}{|c|}{$\begin{array}{l}\text { Speckle noise } \\
(0.5 \%)\end{array}$} & \multicolumn{2}{|c|}{$\begin{array}{l}\text { Speckle noise } \\
(1 \%)\end{array}$} \\
\hline & PSNR & $\mathrm{NC}$ & PSNR & $\mathrm{NC}$ & PSNR & $\mathrm{NC}$ & PSNR & $\mathrm{NC}$ & PSNR & $\mathrm{NC}$ & PSNR & $\mathrm{NC}$ \\
\hline \multicolumn{13}{|c|}{ Boats } \\
\hline 0.01 & 29.98 & 0.6771 & 23.03 & 0.5964 & 28.41 & 0.6962 & 25.21 & 0.6984 & 28.34 & 0.6300 & 25.35 & 0.6087 \\
\hline 0.05 & 29.71 & 0.9383 & 23.00 & 0.8038 & 28.16 & 0.9383 & 25.23 & 0.8778 & 28.19 & 0.8845 & 25.28 & 0.8509 \\
\hline 0.1 & 28.90 & 0.9966 & 22.77 & 0.9249 & 27.83 & 0.9865 & 24.99 & 0.9776 & 27.65 & 0.9899 & 25.01 & 0.9619 \\
\hline 0.15 & 27.87 & 1.0000 & 22.51 & 0.9720 & 26.77 & 1.0000 & 24.69 & 0.9955 & 26.86 & 1.0000 & 24.58 & 0.9877 \\
\hline 0.2 & 26.73 & 1.0000 & 22.11 & 0.9944 & 25.99 & 1.0000 & 24.16 & 0.9978 & 25.93 & 1.0000 & 24.01 & 1.0000 \\
\hline \multicolumn{13}{|l|}{ Baboon } \\
\hline 0.01 & 29.96 & 0.6626 & 23.03 & 0.6020 & 28.59 & 0.7063 & 25.48 & 0.6525 & 27.60 & 0.6312 & 24.61 & 0.6143 \\
\hline 0.05 & 29.77 & 0.9339 & 22.96 & 0.7836 & 28.33 & 0.9159 & 25.27 & 0.8587 & 27.50 & 0.8812 & 24.57 & 0.8173 \\
\hline 0.1 & 29.15 & 0.9955 & 22.82 & 0.9249 & 27.75 & 0.9933 & 25.05 & 0.9619 & 27.16 & 0.9854 & 24.39 & 0.9417 \\
\hline 0.15 & 28.29 & 1.0000 & 22.60 & 0.9630 & 27.09 & 1.0000 & 24.72 & 0.9966 & 26.59 & 0.9966 & 24.09 & 0.9843 \\
\hline 0.2 & 27.31 & 1.0000 & 22.32 & 0.9966 & 26.34 & 1.0000 & 24.34 & 1.0000 & 25.93 & 1.0000 & 23.70 & 0.9966 \\
\hline \multicolumn{13}{|l|}{ Goldhill } \\
\hline 0.01 & 29.98 & 0.6558 & 23.04 & 0.5684 & 28.22 & 0.6704 & 25.49 & 0.6513 & 29.39 & 0.6435 & 26.45 & 0.5953 \\
\hline 0.05 & 29.66 & 0.9182 & 22.95 & 0.7769 & 28.08 & 0.9193 & 25.29 & 0.8442 & 29.12 & 0.9025 & 26.29 & 0.8229 \\
\hline 0.1 & 28.72 & 0.9922 & 22.73 & 0.9025 & 27.62 & 0.9731 & 25.00 & 0.9596 & 28.35 & 0.9865 & 25.86 & 0.9518 \\
\hline 0.15 & 27.53 & 1.0000 & 22.40 & 0.9742 & 26.78 & 0.9955 & 24.36 & 0.9776 & 27.26 & 0.9989 & 25.22 & 0.9944 \\
\hline 0.2 & 26.27 & 1.0000 & 21.94 & 0.9854 & 25.55 & 1.0000 & 23.75 & 0.9978 & 26.09 & 1.0000 & 24.50 & 0.9944 \\
\hline
\end{tabular}


(a)
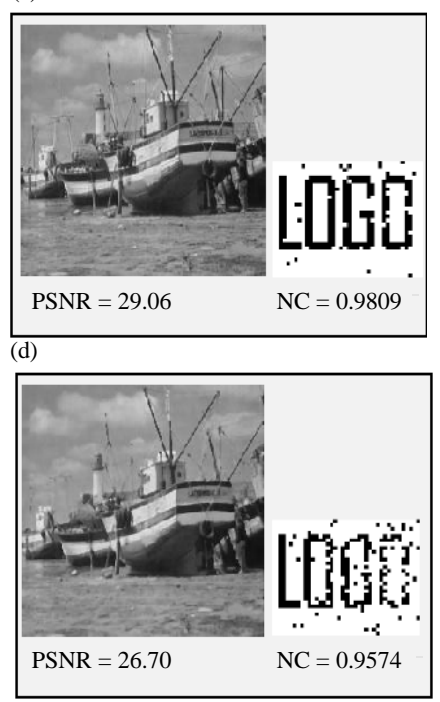

(g)
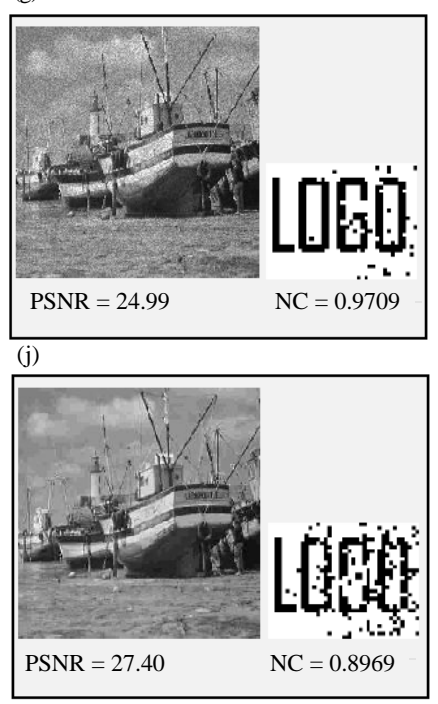

(b)
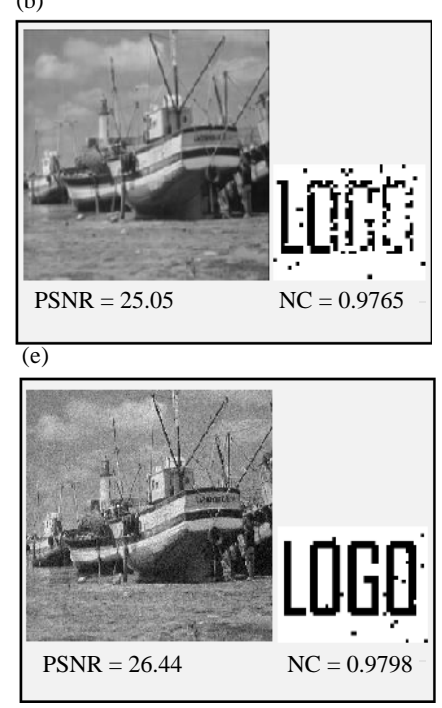

(h)
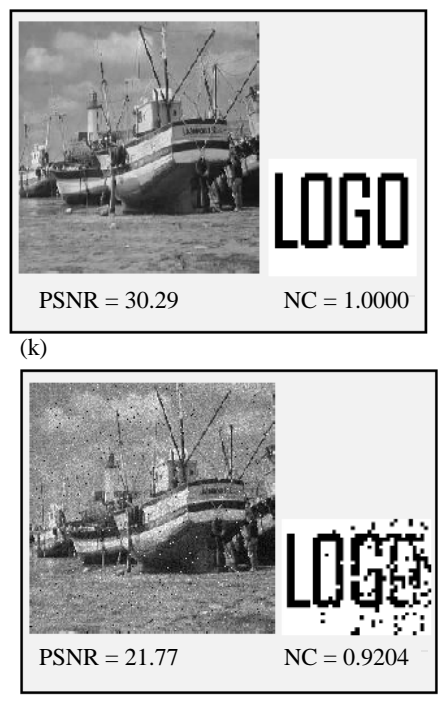

(c)
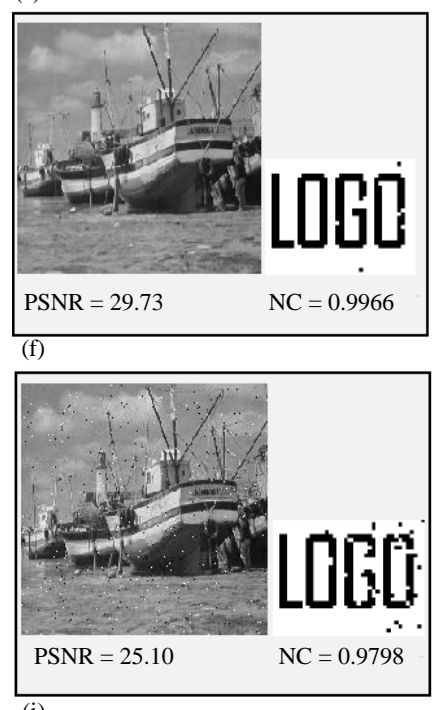

(i)
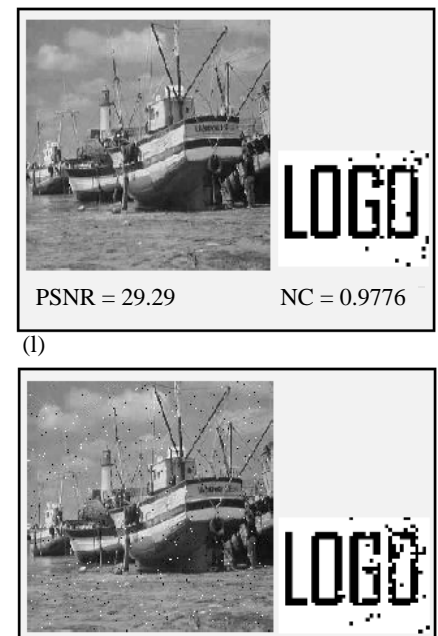

$\mathrm{PSNR}=24.51$

$\mathrm{NC}=0.9664$

Fig. 6: The watermarked boats images subjected to various attacks with the extracted logo watermark images for each: a) Average filter $(3 \times 3)$; b) Average filter $(5 \times 5)$; c) Median filter ( $3 \times 3)$; d) Median filter $(5 \times 5)$; e) Gaussian noise $(\mu=0, v=0.002)$, (f) salt and peppers noise ( $1 \%)$; g) Speckle noise ( $1 \%)$; h) JPEG compression $Q F=30 \%$; i) JPEG compression $\mathrm{QF}=20 \%$; j) JPEG compression $\mathrm{QF}=10 \%$; $\mathrm{k}$ ) Mixed noise: salt and peppers noise (1\%), Gaussian noise $(\mu=0, v=0.001)$, Speckle noise ( $1 \%)$ and 1$) \mathrm{JPEG}$ compression $\mathrm{QF}=50 \%$ with salt and peppers noise $(1 \%)$

Table 3: Performance evaluation and comparison

\begin{tabular}{|c|c|c|c|c|c|c|c|}
\hline \multirow[b]{3}{*}{ Attack } & \multirow[b]{3}{*}{ Parameters } & \multicolumn{6}{|l|}{ Images } \\
\hline & & \multicolumn{2}{|l|}{ Boats } & \multicolumn{2}{|c|}{ Baboon } & \multicolumn{2}{|c|}{ Gold-hill } \\
\hline & & PSNR & $\mathrm{NC}$ & PSNR & $\mathrm{NC}$ & PSNR & $\mathrm{NC}$ \\
\hline No attack & - & 35.51 & 1.0000 & 36.71 & 1.0000 & 33.91 & 1.0000 \\
\hline Median filter & $(3 \times 3)$ & 29.73 & 0.9966 & 22.75 & 0.8890 & 29.97 & 0.9865 \\
\hline Median filter & $(5 \times 5)$ & 26.70 & 0.9574 & 20.40 & 0.8094 & 27.89 & 0.9484 \\
\hline Average filter & $(3 \times 3)$ & 27.95 & 0.9798 & 22.20 & 0.9664 & 28.45 & 0.9675 \\
\hline Average filter & $(5 \times 5)$ & 25.05 & 0.9765 & 20.12 & 0.9608 & 26.13 & 0.9630 \\
\hline Gaussian noise & $(\mu=0, \nu=0.003)$ & 24.84 & 0.9518 & 24.93 & 0.9596 & 24.76 & 0.9406 \\
\hline
\end{tabular}




\begin{tabular}{|c|c|c|c|c|c|c|c|}
\hline \multirow[b]{3}{*}{ Attack } & \multirow[b]{3}{*}{ Parameters } & \multicolumn{6}{|l|}{ Images } \\
\hline & & \multicolumn{2}{|l|}{ Boats } & \multicolumn{2}{|c|}{ Baboon } & \multicolumn{2}{|c|}{ Gold-hill } \\
\hline & & PSNR & $\mathrm{NC}$ & PSNR & $\mathrm{NC}$ & PSNR & $\mathrm{NC}$ \\
\hline Gaussian noise & $(\mu=0, v=0.006)$ & 22.03 & 0.9182 & 22.08 & 0.9114 & 21.97 & 0.8868 \\
\hline Gaussian noise & $(\mu=0, v=0.01)$ & 19.95 & 0.8565 & 19.96 & 0.8397 & 19.92 & 0.8587 \\
\hline Salt and peppers noise & $($ density $=0.01)$ & 25.07 & 0.9720 & 25.10 & 0.9652 & 24.89 & 0.9518 \\
\hline Salt and peppers noise & $($ density $=0.02)$ & 22.25 & 0.9372 & 22.26 & 0.9316 & 22.05 & 0.8957 \\
\hline Salt and peppers & $($ density $=0.03)$ & 20.63 & 0.9036 & 20.49 & 0.9137 & 20.35 & 0.8991 \\
\hline Speckle noise & $($ density $=0.01)$ & 24.99 & 0.9552 & 24.38 & 0.9395 & 25.86 & 0.9585 \\
\hline Speckle noise & $($ density $=0.02)$ & 22.21 & 0.8935 & 21.51 & 0.8756 & 23.21 & 0.9025 \\
\hline Speckle noise & $($ density $=0.03)$ & 20.55 & 0.8677 & 19.86 & 0.8475 & 21.61 & 0.8666 \\
\hline JPEG compression & $(\mathrm{QF}=10)$ & 27.40 & 0.8969 & 22.63 & 0.8823 & 27.61 & 0.8498 \\
\hline JPEG compression & $(\mathrm{QF}=20)$ & 29.29 & 0.9776 & 24.39 & 0.9776 & 29.27 & 0.9686 \\
\hline JPEG compression & $(\mathrm{QF}=30)$ & 30.29 & 1.0000 & 25.53 & 0.9978 & 30.14 & 0.9922 \\
\hline JPEG compression & $(\mathrm{QF}=40)$ & 30.91 & 1.0000 & 26.43 & 0.9989 & 30.69 & 0.9989 \\
\hline JPEG compression & $(\mathrm{QF}=50)$ & 31.37 & 1.0000 & 27.22 & 1.0000 & 31.07 & 1.0000 \\
\hline
\end{tabular}

scheme with varied attacks. Depending on Table 1,2 and Fig. 5, the value of the robustness factor should be carefully selected to maintain the cover image quality and the watermark strength. In this study, the selected value is $(\delta=0.1)$. Figure 5 and 6 show the extracted watermark after subjecting the watermarked boats image to various attacks including JPEG compression, low-pass filtering, and different types of noise. Performance analysis of the proposed scheme is presented in Table 3 using the test images.

\section{CONCLUSION}

An invisible watermarking scheme for grayscale images based on DCT is introduced in this study. The low-frequency region of DCT bands is utilized for embedding a binary watermark image. The watermarking strength is controlled by the user by changing the robustness factor $(\boldsymbol{\delta})$ value according the required robustness level and the characteristics of the host image. The performance of the proposed scheme is evaluated by subjecting the watermarked test images to varied attacks including average filter, median filter, speckle noise, salt and peppers noise, Gaussian noise and JPEG compression with various quality factor. The visual quality of the watermarked image is preserved and can be controlled by the robustness factor $(\boldsymbol{\delta})$ value. Additionally, the extracted watermark holds its high quality in terms of $\mathrm{NC}$ even under certain types of attacks. So, the employing of the low-frequency region of DCT bands improves the performance of the proposed scheme for achieving both the robustness and the imperceptibility even under different attacks.

\section{REFERENCES}

Abdullatif, M., A.M. Zeki, J. Chebil and T.S. Gunawan, 2013. Properties of digital image watermarking. Proceedings of the 2013 IEEE 9th International Colloquium on Signal Processing and its Applications, March 8-10, 2013, IEEE, Kuala Lumpur, Malaysia, ISBN:978-1-4673-5608-4, pp: 207-210.

Chen, W.Y. and S.Y. Huang, 2008. Digital watermarking using DCT transformation. Electron. Eng., 1: 173-184.

Eswaraiah, R., S.A. Edara and E.S. Reddy, 2012. Color image watermarking scheme using DWT and DCT coefficients of R, G and B color components. Intl. J. Comput. Appl., 50: 38-41.

Giri, K.J., M.A. Peer and P. Nagabhushan, 2015. A robust color image watermarking scheme using discrete wavelet transformation. Intl. J. Image Graphics Signal Process., 1: 47-52.

Gunjal, B.L. and S.N. Mali, 2011. Comparative performance analysis of DWT-SVD based color image watermarking technique in YUV, RGB and YIQ color spaces. Intl. J. Comput. Theor. Eng., 3: 714-719.

Huynh-The, T., O. Banos, S. Lee, Y. Yoon and T. Le-Tien, 2016. Improving digital image watermarking by means of optimal channel selection. Expert Syst. Appl., 62: 177-189.

Islam, S. and U.P. Chong, 2014. A digital image watermarking algorithm based on DWT DCT and SVD. Intl. J. Comput. Commun. Eng., 3: 356-360.

Jawad, M.M., E.H. Ali and A.J. Yousif, 2014. A fuzzy random impulse noise detection and reduction method based on noise density estimation. Intl. J. Sci. Eng. Res., 5: 455-468. 
Kumar, A. and Anuradha, 2014. A novel watermarking algorithm for color images based on discrete wavelet transform. Intl. J. Comput. Electr. Eng., 6: 303-306.

Malshe, S., H. Gupta and S. Mandloi, 2012. Survey of digital image watermarking techniques to achieve robustness. Intl. J. Comput. Appl., 45: $1-8$.

Mishra, B.P., H.N. Pratihari and P. Das, 2015. DCT based grey scale still image watermarking using 1-D walsh code and biometric protection. Intl. J. Emerging Trends Technol. Comput. Sci., 4: 28-32.

Parashar, P. and R.K. Singh, 2014. A survey: Digital image watermarking techniques. Intl. J. Signal Image Process. Pattern Recognit., 7: 111-124.

Ram, B., 2013. Digital image watermarking technique using discrete wavelet transform and discrete cosine transform. Intl. J. Advancements Res. Technol., 2: 19-27.
Santhi, V. and A. Thangavelu, 2011. DC coefficients based watermarking technique for color images using singular value decomposition. Intl. J. Comput. Electr. Eng., 3: 8-16.

Singh, R., M. Mathuria, K. Rathore and S. Kumar, 2013. A robust color image watermarking using combination of DWT and DCT. Proceedings of the 4th International Conference on IT Summit Confluence 2013 and the Next Generation Information Technology Summit Confluence, September 26-27, 2013, Institute of Engineering \& Technology Educational, Noida, India, pp: 11-14.

Sinha, M.K., R. Rai and G. Kumar, 2014. Literature survey on digital watermarking. Intl. J. Comput. Sci. Inf. Technol., 5: 6538-6542.

Yusnita, Y. and O.O. Khalifa, 2007. Digital watermarking for digital images using wavelet transform. Proceedings of the IEEE International Conference on Telecommunications and Malaysia International Conference on Communications, May 14-17, 2007, Penang, Malaysia, pp: 665-669. 